\title{
Apert Syndrome (Acrocephalosyndactyly): A Rare Syndromic Craniosynostosis
}

\author{
Shrestha $\mathbf{M}^{1}$, Adhikari N², Shah $\mathbf{G}^{3}$ \\ ${ }^{1}$ Dr. Mahesh Shrestha, Resident MD Paediatrics, National Academy of Medical Sciences, Bir Hospital, ${ }^{2}$ Prof Dr. \\ Neelam Adhikari, Chief, Department of Paediatrics, Patan Hospital, ${ }^{3}$ Dr. Ganesh Shah; Senior Paediatrician, Patan \\ Hospital. Kathmandu, Nepal.
}

Address for correspondence: Dr. Neelam Adhikari, E-mail: neelamadhikari@gmail.com

\begin{abstract}
We report a premature infant who had multisutural craniosynostosis with pointed head, syndactyly of $2^{\text {nd }}$, $3^{\text {rd }}$ and $4^{\text {th }}$ fingers of all four limbs and other findings that were consistent with Apert syndrome. This is perhaps the first case of Apert Syndrome reported from Nepal.
\end{abstract}

Key words: Apert syndrome, craniosynostosis, syndactyly

\section{Introduction}

$\mathrm{C}$ raniosynostosis is defined as a premature fusion of the cranial sutures. It is called primary when there is closure of one or more sutures due to abnormalities of skull development and secondary when there is failure of brain growth and expansion. Most of the primary craniosynostosis presents as an isolated type whereas $10-20 \%$ of them present with syndromes ${ }^{1}$. One of the most familiar and well described form of these syndromic craniosynostosis is Apert Syndrome.

Apert Syndrome or acrocephalosyndactyly is a very rare type of craniosynostosis occurring in approximately 1 per 160,000 to 1 per 200,000 live births $^{2}$. The condition was first reported by Wheaton in 1894. In 1906, Eugène Apert, a French physician, first described nine people with a very similar disorder and in 1920, Park and Powers published an essay on this entity ${ }^{3}$.

\section{Case report}

A preterm 36 weeks, appropriate for gestational age female baby with birth weight of $2500 \mathrm{~g}$ was delivered vaginally to a primigravida. Apgar scores at 1 and 5 minutes were 7 and 8 respectively. Mother had attended Patan hospital for antenatal visits regularly. Results of ultrasound scanning performed in the third trimester had been reported as normal and consistent with dates. Initial evaluation revealed a mildly tachypneic baby with craniosynostosis of all the sutures except the metopic suture, syndactyly in all the four limbs, proptosis and bilateral choanal atresia (unable to pass nasogastic tube through the nose).

On further evaluation, the baby had closure of all sutures and fontanelles except for the metopic suture which gave a 'pointed' head-like appearance (Greek: 'acro' means 'pointed', hence the term acrocephalosyndactyly). The syndactyly was present in $2^{\text {nd }}, 3^{\text {rd }}$ and $4^{\text {th }}$ fingers in all the four limbs (see fig 1). Hypertelorism was present, proptosis was mild in nature. Nasal bridge was depressed (see fig 2). Choanal atresia was bilateral. The child had normal oral cavity, genitalia and spine. No esophageal atresia was present as red rubber catheter could be passed easily. Vitals except for a mild tachypnoea were stable. Systemic examination was normal. All the findings were consistent with Apert syndrome. Parents were informed and counseled.

The child remained tachypneic and developed chest retractions at 20 hours of life. Septic workup was done and empirical antibiotics were started. The child also became icteric and required phototherapy at 44 hours of life. Mother's blood group was $\mathrm{O}$ positive. The condition of the child had been explained to the parents every day. The child had slow improvement in respiratory distress and hyperbilirubinemia in the next few days. However at $7^{\text {th }}$ day of life, the father took the child to Hospital for Disabled Children for consultation. 


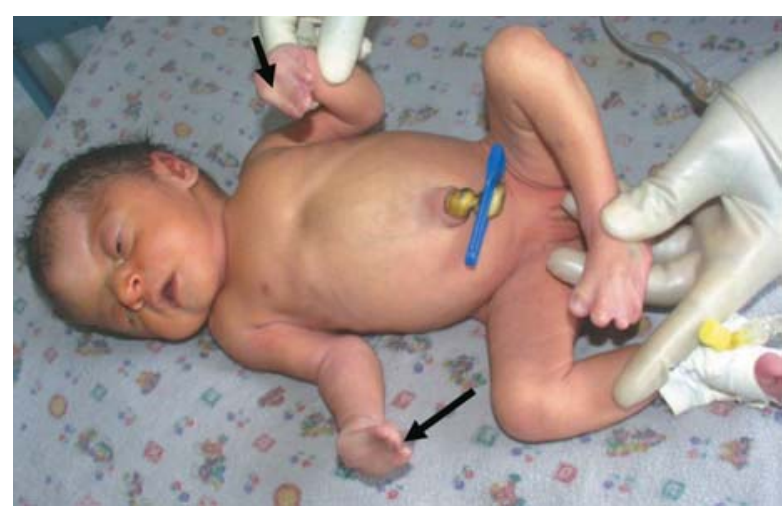

Fig 1: Showing fused $2^{\text {nd }}, 3^{\text {rd }}$ and $4^{\text {th }}$ fingers in all the limbs (see black arrows)

\section{Discussion}

Apert syndrome is a very rare syndrome and considering the total annual patients of our hospital, we would be seeing probably 1 case in 3 or 4 years. But since the features of Apert syndrome are very characteristic especially syndactyly of $2^{\text {nd }}, 3^{\text {rd }}$ and $4^{\text {th }}$ fingers and pointed head like appearance, a careful observation would lead to diagnoses of this rare condition. Apart from the features mentioned above, there can be other abnormalities in varying degrees of proportion. Deceleration of linear growth after birth, progressive hydrocephalus, agenesis of corpus callosum, ventriculomegaly, mental retardation, delayed eruption of teeth, fusion of cervical vertebrae, acne in adolescence and gastrointestinal, respiratory, cardiac and genitourinary anomalies may occur ${ }^{4}$.

Most cases of Apert syndrome are sporadic although autosomal dominant inheritance may occur. Mutations in fibroblast growth factor receptor 2 gene (FGFR2) that maps to chromosome 10q25-10q26 have been shown to be associated with syndromic craniosynostosis like Apert syndrome ${ }^{5}$.

The important differential diagnosis of Apert syndrome would be other similar syndromes with craniosynostosis, like Crouzon syndrome, Carpenter syndrome, Chotzen syndrome and Pfeiffer syndrome. Crouzon syndrome is very similar to Apert syndrome but the proptosis is very marked and syndactyly of $2^{\text {nd }}$, $3^{\text {rd }}$ and $4^{\text {th }}$ fingers is absent. Carpenter syndrome occurs as an autosomal recessive condition, and the many fusions of sutures tend to produce the kleeblattschädel skull deformity. Chotzen syndrome is characterized by asymmetric craniosynostosis and plagiocephaly. Pfeiffer syndrome most often presents with turricephaly. The eyes are prominent and widely spaced, and the thumbs and great toes are short and broad.

Treatment for Apert syndrome is surgical. Early surgery before one year is indicated. It has to be done

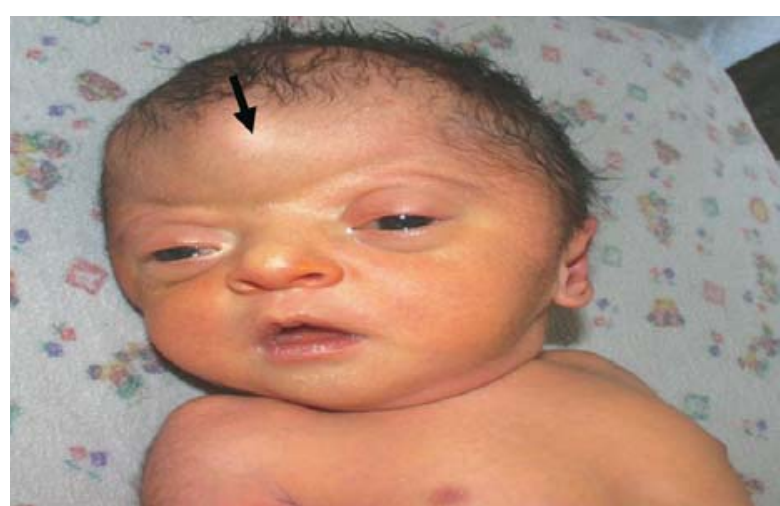

Fig 2: Showing patient with multisutural craniosynostosis with pointed head or acrocephaly (black arrow), depressed nasal bridge and hypertelorism.

even earlier if there is evidence of increased intracranial pressure. Presence of upper and/or lower airway compromise should warrant early management to prevent early death ${ }^{6}$. Surgery for release of syndactyly is done in stages. Examples of good outcome have been reported in literature.

\section{Acknowledgement}

We would like to thank Mr. Macha B. Shakya, librarian of Patan Hospital for proving us the photos of the child for this case report.

Funding: None.

Competing interest: None.

\section{References}

1. Kinsman SL, Johnston MV- Craniosynostosis. In: Kliegman RM, Behrman RE, Jenson HB, Stanton BF - Nelson Textbook of Pediatrics, Vol 2, $18^{\text {th }}$ ed. Philadelphia: Saunders, 2007: 2455-2456.

2. Kaplan LC. Clinical Assessment and Multispecialty Management of Apert Syndrome. Clin Plast Surg April 1991:18(2); 217-25.

3. Apert Syndrome. In: Smith's Recognizable Patterns of Human malformations, 5th edition, 418-419.

4. Cohen MM, Kreiborg S Jr: The Central Nervous System in the Apert Syndrome. Am J Med. Genet. 1990 Jan; 35(1):36-45.

5. Wilkie, A.O.M. et al: Apert Syndrome results from Localized Mutations Of FGFR2 and is Allelic with Crouzon Syndrome. Nat. Genet. Feb 1995; 9(2):165-172.

6. Sloan GM,WellsKC, RaffleC, Gordon McComb J: Surgical Treatment of Craniosynostosis: Outcome Analysis of 250 Consecutive Patients. Pediatrics Jul 1997; 100(1):E2 